\title{
3 Research Square \\ Potential Role of CTNNA3 and FRMPD4 in Vascular Thrombosis of Colon Adenocarcinoma
}

\section{Bin Liu}

The Second People's Hospital of Deyang City

\section{Yu Xiang}

Suining Central Hospital

Weihua Guo ( $\sim$ gwh919773015@163.com )

Panzhihua Municipal Central Hospital

\section{Research Article}

Keywords: Colon adenocarcinoma, Tumor thrombus, Biomarkers, CTNNA3, FRMPD4

Posted Date: December 14th, 2021

DOI: https://doi.org/10.21203/rs.3.rs-1160821/v1

License: (c) (1) This work is licensed under a Creative Commons Attribution 4.0 International License. Read Full License 


\section{Abstract}

Background: Vascular tumor thrombus is an important pathological feature of malignant tumors, closely related to lymph node metastasis, and considered to be tumor micrometastasis. Two down-regulated genes, Catenin Alpha 3 (CTNNA3) and FERM And PDZ Domain Containing 4 (FRMPD4) were selected via analyzing the differential expression of vascular tumor thrombus in colon adenocarcinoma and paracancerous tissues. Furthermore, we observed the potential role of them in vascular thrombosis of colon adenocarcinoma.

Method: Candidate genes for vascular thrombosis of colon adenocarcinoma were screened by GSE127069, and then pan-cancer verification and immune infiltration analysis were performed. The relationship between gene and vascular thrombosis was analyzed from the level of gene mutation by cBioPortal. Finally, the clinical samples collected were used for expression verification.

Results: CTNNA3 and FRMPD4 were low-expressed in vascular tumor thrombus of colon adenocarcinoma, which were positively correlated with microsatellite instability (MSI). They were also closely related to immune microenvironment and immune cell subtype infiltration. According to the analysis of gene mutation, their gene deletion was related to the vascular invasion indicator. Finally, the protein and mRNA expression of CTNNA3 and FRMPD4 were down-regulated in the samples of vascular thrombosis of colon adenocarcinoma, compared with the normal glands which from paracancerous tissues.

Conclusion: Our study demonstrates that CTNNA3 and FRMPD4 may be potential biomarkers of vascular thrombosis in colon adenocarcinoma. These results may provide a new strategy to identify the micrometastasis in colon adenocarcinoma.

\section{Introduction}

Colorectal cancer is the third most common cancer in men worldwide, after prostate cancer and lung cancer, and the second most common cancer in women, after breast cancer (1). In recent years, the incidence of colon cancer has been an increasing trend, and most of patients are diagnosed in the middle or advanced stages (2). Although postoperative overall survival of colon cancer has been prolonged in recent years, however, there are still $30 \% \sim 50 \%$ of patients with postoperative recurrence, tumor metastasis, and eventually death (3).

The invasion and metastasis of cancer cells are important factors that determine the prognosis of patients with colon cancer, and the occurrence of tumor thrombus in blood vessels is a prerequisite for tumor invasion of vascular system and lymph node metastasis $(4,5)$. Vascular tumor thrombus infiltration is an important prognostic factor for many malignant tumors, such as endometrial cancer (6), breast cancer (7), gastric cancer and colon cancer (8). Different studies have suggested that the incidence of tumor thrombus in the midvein of colon cancer ranges from $10 \%$ to $89.5 \%(9-11)$. Colon cancer thrombus mainly includes direct tumor invasion, blood reflux, tumor cell exfoliation, adhesion, 
infiltration, proliferation (12). The cancer cells can infiltrate into submucosa which is rich in vascular system, easily infiltrate and form vascular tumor thrombus. Genetic mutations are critical tovascular tumor thrombus in colon cancer (13). However, the relation between gene alteration and tumor thrombus is not clear. Therefore, it is of great significance to find potential biomarkers of vascular thrombosis for treating colon cancer.

Vascular tumor thrombus leads tumor cells to better tolerate injury, promote tumor cell metastasis (14). Study has shown that, compared with the negative group, the risk of death in the vascular tumor thrombus positive group increased by 1.748 times, and OS significantly decreased (15). Lymphatic tumor thrombus infiltration is an important risk factor for sentinel lymph node metastasis (16). Therefore, patients with II stage of colon cancer with positive vascular tumor thrombus infiltration should receive chemotherapy even if there is no lymph node metastasis after operation. it also has guiding value for preoperative neoadjuvant therapy.

Antivascular and antilymphatic therapy is of great significance for patients with vascular tumor thrombus, such as bevacizumab and Shunitinib can inhibit angiogenesis, and reduce lymph node metastasis $(17,18)$. At present, the common method to detect vascular tumor thrombus is tissue section hematoxylin-eosin (HE) staining (19), but the detection rate of vascular tumor thrombus in II colorectal cancer varies from $5.2 \%$ to $30 \%$ (20). Unfortunately, the ability to distinguish lymphovascular invasion from blood vessel invasion is poor.

In this study, two significantly low-expressed genes Catenin Alpha 3 (CTNNA3) and FERM And PDZ Domain Containing 4 (FRMPD4) were obtained from the analysis of the difference between intratumoral tumor thrombus group and normal adjacent tissues of colon adenocarcinoma. After TCGA pan-cancer verification, it was found that these two genes may be associated with microsatellite instability (MSI) and immune infiltration. According to the analysis of gene mutations, we found two genes had different degrees of gene mutation, such as amplification, deep deletion and missense mutation, and were related to methylation. Immune cell infiltration analysis also shown that these two genes were associated with different immune cell infiltration. Finally, we found that these two genes were down-regulated in patients with colon adenocarcinoma thrombus at the mRNA and protein levels. In short, we found potential biomarkers in patients with colon adenocarcinoma thrombus, which has a certain guiding significance for the therapy of patients with cancer thrombus.

\section{Material And Methods}

\subsection{Patient Data acquisition}

RNA sequencing (GSE118389) for colon adenocarcinoma tissues and adjacent tissues of third-stage colon cancer patients with blood vascular thrombus was downloaded from GEO (Gene Expression Omnibus) (https://www.ncbi.nlm.nih.gov/geo/). Twenty cases of colon adenocarcinoma tumor thrombus and adjacent normal tissues came from the Second People's Hospital of Deyang City for detection of gene expression. The patients with left colon cancer included 9 males and 11 females. The clinical 
stages were II stage $(n=15)$ and III stage $(n=5)$. The pathological types of the samples were all tubular adenocarcinoma. This study has been approved by the hospital ethics committee and the patient's informed consent.

\subsection{Differentially Expressed genes}

'Limma' package was used to perform the batch correction of the GSE118389 database. The R software (version 4.0.5) was used to perform differentially expressed genes in the normal groups $(n=3)$ and colon cancer thrombus $(n=3)$ groups. The cutoff criteria were FDR $p$-value $<0.05$ and $\operatorname{logFC}>1$.

\subsection{Gene Ontology (GO) and Kyoto Encyclopedia of Genes and Genomes (KEGG) pathway enrichment analysis}

R software were used to perform GO and KEGG analysis. 88 differentially expressed genes and logFC values were selected for functional enrichment analysis of GO and KEGG.

\subsection{Protein-protein interaction}

The protein-protein interaction (PPI) network was analyzed online through STRING website (https://string$\mathrm{db}$. org/). The co-expression relationship of 88 differential genes was visualized by PPI network.

\subsection{Association between gene expression and MSI in pan-cancer}

The MSI score was obtained from TCGA. Spearman's method was employed to evaluate the correlation between cancer gene expression and MSI. The radar map was designed using the ' $f m s b$ ' package from R software to visualize the both indicators.

\subsection{Correlation of gene expression with tumor immune microenvironment and immune cell infiltration}

The ESTIMATE algorithm ("estimate" and "limma" packages in R software) was employed to calculate immune scores, for predicting the tumor purity and infiltrating immune cells in colon adenocarcinoma. The "ggplot2", "ggpubr" and "ggExtra" packages in R software were employed to evaluate the correlation between CTNNA3 or FRPMD4 mRNA expression and the tumor immune microenvironment.

\subsection{Correlation analysis of immune cell infiltration}

The Pearson' method was used to analysis the correlation between CTNNA3 or FRPMD4 mRNA expression and immune cell infiltration. The infiltration data of B cells, CD $4+T$ cells, CD8 + T cells, dendritic cells, macrophages, neutrophils, T cell regulatory (Tregs) can be downloaded from the TIMER 2.0 database (http://timer.cistrome.org/).

\section{8 cBioPortal online analysis}

Perform gene mining and analysis on the cBioPortal (https://www.cbioportal.org/), and select the TCGA colon adenocarcinoma database for "Explore Selected Studies". Then click the "OncoPrint", "Plots", 
"Mutations", "Genomics Alterations" modules to perform gene mining analysis.

\subsection{Immunohistochemistry (IHC).}

Human colon adenocarcinoma samples, colon adenocarcinoma thrombus, and adjacent normal tissue were used for IHC staining. IHC was performed according to the manufacturer's instructions (cat. no. SP9001; ZSGB-BIO). The primary antibody (anti-FRMPD, ab113420, abcam), anti-CTNNA3 (ab184916, abcam), and anti-CD34 (ab81289, abcam)) were used for IHC. The specific operation steps were performed as previously described (21). The protein expression level was determined according to the staining intensity and the percentage of immunoreactive cells.

\subsection{Quantitative Real Time PCR.}

RNA extraction, reverse transcription, and PCR amplification were conducted according to the manufacturer's instructions (Takara Bio, Inc.). The specific operation steps were performed as previously described (22). The primer sequences were as follows: GAPDH sense, 5'-CAATGACCCCTTCATTGACC-3' and antisense, 5'-GACAAGCTTCCCGTTCTCAG-3'; CTNNA3 sense, 5'- TTTCTTTGCTGAGCCTCGTCTG-3' and antisense, 5'- GGTCCAAACATTCACCGTGGAG-3'. FRMPD4 sense, 5'-

AAGCCTCTTGAGAGAACGTGTCAG-3' and antisense, 5'- ATGGAGGAGGACCTTGAAGGAG-3'. All reactions were performed in triplicate. The relative expression of gene to GAPDH (CTNNA3/GAPDH or FRMPD4/GAPDH) was calculated using the $2-\Delta \Delta$ Cq method.

\subsection{Statistical analysis}

GraphPad Prism 7.0 was used for statistical analysis. Student's t-tests were used to perform differential comparisons of two groups. $P$ value $<0.05$ considered to be statistically significant. The integrated analysis of these data was performed using R software (version 4.0.3).

\section{Results}

\subsection{CTNNA3 and FRMPD4 are low expressed in colon adenocarcinoma tumor thrombus}

The differential analysis of the gene expression profiles of the colon adenocarcinoma para-normal group $(n=3)$ and the colon adenocarcinoma thrombus group $(n=3)$ was performed in the GSE127069 chip. The volcano map (Fig.1A) and the heat map (Figure 1B) were used to visualize differentially expressed genes. The results shown that CTNNA3 and FRMPD4 were specifically down-regulated in colon adenocarcinoma tumor thrombus samples. The protein-protein interaction network also visualized the co-expression of differential genes (Fig. 2). According to the analysis of the number of PPI nodes, the results shown that the number of CTNNA3 and FRMPD4 nodes was the most (Fig. 1C). In addition, GO and KEGG enrichment analysis were performed on 88 differential expressed genes (Fig. 1D, E), GO analysis shown that the differential genes were related to second-messenger-mediated signaling, positive regulation of cytosolic calcium ion concentration, regulation of cytosolic calcium ion concentration, cellular calcium ion homeostasis, and calcium ion homeostasis. KEGG analysis shown that the 
differential genes are related to chemokine signaling pathway, cocaine addiction, amphetamine addiction, gastric acid secretion, and insulin secretion.

\subsection{CTNNA3 and FRMPD4 are related to MSI and immune microenvironment.}

In order to further verify the expression and function of CTNNA3 and FRMPD4 in colon adenocarcinoma, we performed pan-cancer analysis on the TCGA database. The results shown that the expression of CTNNA3 and FRMPD4 in colon adenocarcinoma was lower than that in normal tissues (Fig. 3A, C), but there was no significant difference in their expression between tumor stages (Fig. 3B, D). MSI is caused by a defect in the mismatch repair gene and is strongly correlated with the occurrence of tumors. Clinically, MSI has been used as an important molecular marker for the prognosis of colorectal cancer and the formulation of adjuvant treatment programs. We found that CTNNA3 and FRMPD4 were positively correlated with MSI in colon adenocarcinoma (Fig. 3E, F). Due to the tumor immune microenvironment, the heterogeneity between tumor cells is activated, thereby increasing multidrug resistance, causing tumor cell progression and metastasis. Therefore, it is reasonable to explore the correlation between tumor immune microenvironment and the expression of CTNNA3 or FRMPD4 in colon adenocarcinoma. The ESTIMATE algorithm was used to calculate immune cell scores for colon adenocarcinoma. The results shown that the expression of CTNNA3 or FRMPD4 were positively related to the immune scores in colon adenocarcinoma (Fig. 3G, H), indicating that the level of CTNNA3 and FRMPD4 expression was upgraded as immune cells increases.

\subsection{CTNNA3 and FRMPD4 are related to the infiltration of immune cell subtypes.}

For analyzing the relationship between the expression of CTNNA3 or FRMPD4 and immune cell infiltration in colon adenocarcinoma, The correlation between CTNNA3 or FRMPD4 and immune cell subtype infiltration was evaluated by using TIMER 2.0. The results shown that the correlation coefficients between Purity, B cells, CD4 + T cells, CD8 + T cells, DCs, macrophages, neutrophils, and Tregs, and CTNNA3 were $-0.152,0.005,0.22,0.044,0.224,0.27,0.207$, and 0.031 , respectively (Fig. 4A, $P<0.001$ ). And the correlation coefficients between Purity, B cells, CD $4+T$ cells, CD $8+T$ cells, DCs, macrophages, neutrophils, and Tregs, and FRMPD4 were $-0.132,-0.207,0.317,0.098,0.364,0.318,0.311$, and -0.011 , respectively (Fig. 4B, $\mathrm{P}<0.001$ ).

\subsection{Gene mutations of CTNNA3 and FRMPD4 are associated with vascular invasion indicator}

In order to further explore the functions of CTNNA3 and FRMPD4 in colon adenocarcinoma thrombus, we used the cBioPortal online tool to analyze gene functions. As shown in Fig. 5A and 6A, the genetic changes of CTNNA3 or FRMPD4 and the relationship between vascular invasion indicator were shown respectively. The results indicated that missense mutations of CTNNA3 were related to vascular invasion indicator, and missense mutations and deep deletion of FRMPD4 were related to vascular invasion indicator. Next, we analyzed the genes related to genetic altered, and the results shown that the genetic altered of CTNNA3 were related to TNN, PIK3CA, MACF1, TENM3, and RIMS1 (Fig. 5B), while the genetic altered of FRMPD4 were related to TLR7, FAM9B, WWC3, MXRA5, and DMD related (Fig. 6B). The 
analysis of correlation between gene mRNA expression and copy number variation shown that the mRNA expression of CTNNA3 and FRMPD4 were all related to shallow deletion, diploid, gain, etc. (Fig. 5C, Fig. 6C). Interestingly, the mRNA expression of CTNNA3 was also related to gene methylation (HM450) (Fig. $5 D$, Spearman, $R=0.34$; Pearson, $R=0.42$. $P<0.001$ ). The mutation site analysis results shown that the mutation of CTNNA3 The site was located at Y414C (Fig. 5E), and the mutation site of FRMPD4 was located at E619A/D (Fig. 6D).

\subsection{CTNNA3 and FRMPD4 were down-regulated in patients with colon adenocarcinoma thrombus}

Finally, in order to validate the expression of CTNNA3 and FRMPD4 in clinical practice, we collected 20 patients with colon adenocarcinoma thrombus. HE staining and CD34 staining were used to determine the morphology of tumor tissue and tumor thrombus, respectively (Fig. 7A). The expression of CTNNA3 and FRMPD4 in tumor thrombus tissues was lower than that in normal tissues (Fig. 7A, D, E). We also performed gene mRNA expression verification, which shown that the expression of CTNNA3 and FRMPD4 mRNA in colon adenocarcinoma embolus was lower than that in normal adjacent tissues (Fig. 7B, C).

\section{Discussion}

In this study, we analyzed the differential expression profiles of genes from GSE127069 and selected biomarkers for vascular tumor thrombus of colon adenocarcinoma. Then the gene function was further explored in TCGA and cBioPortal databases. Finally, the gene expression was verified with the collected clinical samples. These results suggested that CTNNA3 and FRMPD4 might exist as biomarkers of vascular tumor thrombus in colon adenocarcinoma. Vascular tumor thrombus is one of the characteristics of tumor cells invading adjacent blood vessels and lymphatic vessels, which can be regarded as the early manifestation of lymph node metastasis (23). Both domestic and foreign guidelines regarded vascular tumor thrombus positive as one of the items to evaluate the risk factors of colorectal cancer $(24,25)$.

Vascular tumor thrombus is not only an indicator of poor prognosis, but also a manifestation of micrometastasis (11). Related studies have confirmed that vascular invasion is related to the depth of tumor invasion and lymph node metastasis (26). We found that CTNNA3 and FRMPD4 were downregulated in vascular thrombosis of colon cancer and were related to calcium metabolism. Calcium metabolism is closely related to thrombosis (27), which may be one of the reasons why CTNNA3 and FRMPD4 become potential biomarkers of vascular thrombosis in colon adenocarcinoma.

MSI, a class of short tandem repeat DNA sequences in the genome, is generally composed of 1-6 nucleotides and is arranged in tandem repeats. Due to the difference in the number of core repeat units, MSI have population polymorphism. Popat S, et al (28) published meta-analysis of 7642 patients with II colorectal cancer in 32 studies shown that compared with MSS patients, the risk of death in MSI-H patients was $0.65(95 \% \mathrm{Cl}, 0.59-0.71)$, a reduction of up to $35 \%$. At present, there is a great deal of evidence that MSI-H is a good marker for the prognosis of patients with stage II colorectal cancer (29). It has also been found that tumors with MSI-H typing have high immune cell infiltration against new 
antigens, but are offset by immune checkpoint suppressor ligands such as PD-L1, which binding to PD-1 prevents T cell activation (30). Recently, it has been reported that immune checkpoint inhibitor (pembrolizumab) was effective in MSI-H patients with tumor thrombosis. This reflects the relationship between tumor thrombus and MSI and tumor immune microenvironment (31). Consistent with the previously studies, we found that CTNNA3 and FRMPD4 were positively correlated with MSI, as well as with immune microenvironment and immune cell subtype infiltration.

However, due to the difference in the detection technology of vascular cancer thrombus, the detection rate of vascular cancer thrombus is quite different, resulting in many false negative, which may weaken the value of vascular cancer thrombus, resulting in a lack of understanding of vascular cancer thrombus. The study of 75 cases of colorectal cancer found that the lymphatic infiltration rate of HE staining was $19 \%$, while the detection rate of lymphatic invasion of monoclonal antibody D2-40 was $40 \%$ ( $P<$ 0.001) (32). Morgantetti G, et al (33) also believes that CD34 antibody has strong stability, sensitivity and repeatability in the detection of capillaries and small vascular endothelial cells in tumors, so CD34 antibody is the preferred vascular endothelial cell marker for the evaluation of vascular cancer thrombus. We also used CD34 as a marker of vascular thrombus when we verified the protein expression by immunohistochemistry, which further verified the expression of candidate genes in vascular tumor thrombus.

We further found that both CTNNA3 and FRMPD4 have a high proportion of gene deep deletion in gene mutations in colon adenocarcinoma. That also indicated that downregulation of CTNNA3 and FRMPD4 in vascular tumor thrombus of colon adenocarcinoma is understandable. CTNNA3 is a tumor suppressor, which often mutates in laryngeal carcinoma and is downregulation in bladder urothelial carcinoma (34). CTNNA3 is a tumor suppressor of hepatocellular carcinoma, which inhibits the proliferation, migration and invasion of hepatocellular carcinoma. CTNNA3 inhibits AKT signal, which in turn reduces PCNA and MMP-9 in tumor cells (35). There are few studies on FRMPD4 in tumors. FRMPD4 plays an important role in normal cognitive development and function in humans and mice, and FRMPD4 mutation may lead to $X$-linked mental retardation syndrome by destroying the morphology of dendritic spines in glutamatergic neurons (36). The above studies and results shown that CTNNA3 and FRMPD4 have the characteristics of biomarkers in vascular tumor thrombus of colon adenocarcinoma.

The advantage of this study is that multiple databases are used to analyze the selected candidate genes, and clinical samples are used to verify the results. Then, this study also has many limitations. For example, there are few clinical samples for verification, and the candidate genes have not been verified from the biological function level. In the future research, we will reasonably design the experimental scheme to further study the candidate genes.

\section{Conclusion}


In our study, we performed integrated analysis of the GEO, TCGA and cBioPortal databases and found that CTNNA3 and FRMPD4 are involved in cancer biological functions such as MSI, immune subtype infiltration, and methylation in colon adenocarcinoma, and their mutations are also associated with vascular invasion indicator. Viewed in total, CTNNA3 and FRMPD4 may be regarded as potential biomarkers in patients with colon adenocarcinoma thrombus.

\section{Declarations}

\section{Funding}

No funding information.

\section{Consent for publication}

The patient consented all the individual person's data to publish.

\section{Acknowledgments}

We thank the TCGA and GSE118389 for sharing large amounts of data.

\section{Availability of data and materials}

GSE118389 was downloaded from GEO (Gene Expression Omnibus)

(https://www.ncbi.nlm.nih.gov/geo/). And TCGA datasets were visualized in cBioPortal (https://www.cbioportal.org/).

\section{Authors' contributions}

$\mathrm{BL}$ provided the human tissue, designed and performed the experiments, analyzed the data, and wrote the manuscript. YX analyzed the data and prepared figures. WG guided the design of experiments and drafted the manuscript. All authors read and approved the final manuscript.

\section{Ethics approval and consent to participate}

Ethics approval and protocols were approved by the Ethics approval of the Second People's Hospital of Deyang City. The patient provided informed consent for the publication.

\section{Competing interests}

There is no conflict among all of the authors.

\section{References}

1. Ferlay J, Colombet M, Soerjomataram I, Mathers C, Parkin DM, Pineros M, et al. Estimating the global cancer incidence and mortality in 2018: GLOBOCAN sources and methods. International journal of 
cancer. 2019;144(8):1941-53.

2. Bray F, Ferlay J, Soerjomataram I, Siegel RL, Torre LA, Jemal A. Global cancer statistics 2018: GLOBOCAN estimates of incidence and mortality worldwide for 36 cancers in 185 countries. CA Cancer J Clin. 2018;68(6):394-424.

3. Lieberman DA, Rex DK, Winawer SJ, Giardiello FM, Johnson DA, Levin TR. Guidelines for colonoscopy surveillance after screening and polypectomy: a consensus update by the US MultiSociety Task Force on Colorectal Cancer. Gastroenterology. 2012;143(3):844-57.

4. Qi LN, Ma L, Wu FX, Chen YY, Xing WT, Jiang ZJ, et al. S100P as a novel biomarker of microvascular invasion and portal vein tumor thrombus in hepatocellular carcinoma. Hepatol Int. 2021;15(1):11426.

5. Tilki D, Nguyen HG, Dall'Era MA, Bertini R, Carballido JA, Chromecki T, et al. Impact of histologic subtype on cancer-specific survival in patients with renal cell carcinoma and tumor thrombus. Eur Urol. 2014;66(3):577-83.

6. Sadozye AH, Harrand RL, Reed NS. Lymphovascular Space Invasion as a Risk Factor in Early Endometrial Cancer. Curr Oncol Rep. 2016;18(4):24.

7. Snoderly HT, Boone BA, Bennewitz MF. Neutrophil extracellular traps in breast cancer and beyond: current perspectives on NET stimuli, thrombosis and metastasis, and clinical utility for diagnosis and treatment. Breast cancer research : BCR. 2019;21(1):145.

8. Zheng Z, Zhang Y, Zhang L, Li Z, Wu X, Liu Y, et al. A nomogram for predicting the likelihood of lymph node metastasis in early gastric patients. BMC cancer. 2016;16:92.

9. Abdulkader M, Abdulla K, Rakha E, Kaye P. Routine elastic staining assists detection of vascular invasion in colorectal cancer. Histopathology. 2006;49(5):487-92.

10. Lim SB, Yu CS, Jang SJ, Kim TW, Kim JH, Kim JC. Prognostic significance of lymphovascular invasion in sporadic colorectal cancer. Dis Colon Rectum. 2010;53(4):377-84.

11. Betge J, Pollheimer MJ, Lindtner RA, Kornprat P, Schlemmer A, Rehak P, et al. Intramural and extramural vascular invasion in colorectal cancer: prognostic significance and quality of pathology reporting. Cancer. 2012;118(3):628-38.

12. Quencer KB, Friedman T, Sheth R, Oklu R. Tumor thrombus: incidence, imaging, prognosis and treatment. Cardiovasc Diagn Ther. 2017;7(Suppl 3):S165-S77.

13. Song Y, Wang L, Ran W, Li G, Xiao Y, Wang X, et al. Effect of Tumor Location on Clinicopathological and Molecular Markers in Colorectal Cancer in Eastern China Patients: An Analysis of 2,356 Cases. Front Genet. 2020;11:96.

14. Amri R, England J, Bordeianou LG, Berger DL. Risk Stratification in Patients with Stage II Colon Cancer. Ann Surg Oncol. 2016;23(12):3907-14.

15. Zhong JW, Yang SX, Chen RP, Zhou YH, Ye MS, Miao L, et al. Prognostic Value of Lymphovascular Invasion in Patients with Stage III Colorectal Cancer: A Retrospective Study. Med Sci Monit. 2019;25:6043-50. 
16. La Verde N, Biagioli E, Gerardi C, Cordovana A, Casiraghi C, Floriani I, et al. Role of patient and tumor characteristics in sentinel lymph node metastasis in patients with luminal early breast cancer: an observational study. Springerplus. 2016;5:114.

17. Cunningham D, Lang I, Marcuello E, Lorusso V, Ocvirk J, Shin DB, et al. Bevacizumab plus capecitabine versus capecitabine alone in elderly patients with previously untreated metastatic colorectal cancer (AVEX): an open-label, randomised phase 3 trial. Lancet Oncol. 2013;14(11):107785 .

18. Kodera Y, Katanasaka Y, Kitamura Y, Tsuda H, Nishio K, Tamura T, et al. Sunitinib inhibits lymphatic endothelial cell functions and lymph node metastasis in a breast cancer model through inhibition of vascular endothelial growth factor receptor 3. Breast cancer research : BCR. 2011;13(3):R66.

19. Fu CH, Chen CH, Lin YH, Lee CW, Tsai LK, Tang SC, et al. Fibrin and Platelet-Rich Composition in Retrieved Thrombi Hallmarks Stroke With Active Cancer. Stroke. 2020;51(12):3723-7.

20. Yuan $H$, Dong Q, Zheng $B, H u X, X u$ JB, Tu S. Lymphovascular invasion is a high risk factor for stage I/II colorectal cancer: a systematic review and meta-analysis. Oncotarget. 2017;8(28):46565-79.

21. Fu R, Han CF, Ni T, Di L, Liu LJ, Lv WC, et al. A ZEB1/p53 signaling axis in stromal fibroblasts promotes mammary epithelial tumours. Nat Commun. 2019;10(1):3210.

22. Cortes M, Sanchez-Moral L, de Barrios O, Fernandez-Acenero MJ, Martinez-Campanario MC, EsteveCodina A, et al. Tumor-associated macrophages (TAMs) depend on ZEB1 for their cancer-promoting roles. EMBO J. 2017;36(22):3336-55.

23. Skancke M, Arnott SM, Amdur RL, Siegel RS, Obias VJ, Umapathi BA. Lymphovascular Invasion and Perineural Invasion Negatively Impact Overall Survival for Stage II Adenocarcinoma of the Colon. Dis Colon Rectum. 2019;62(2):181-8.

24. Benson AB, Venook AP, Al-Hawary MM, Cederquist L, Chen YJ, Ciombor KK, et al. NCCN Guidelines Insights: Colon Cancer, Version 2.2018. J Natl Compr Canc Netw. 2018;16(4):359-69.

25. National Health Commission of the People's Republic of C. [Chinese Protocol of Diagnosis and Treatment of Colorectal Cancer (2020 edition)]. Zhonghua Wai Ke Za Zhi. 2020;58(8):561-85.

26. Al-Sukhni E, Attwood K, Gabriel EM, LeVea CM, Kanehira K, Nurkin SJ. Lymphovascular and perineural invasion are associated with poor prognostic features and outcomes in colorectal cancer: A retrospective cohort study. Int J Surg. 2017;37:42-9.

27. Volz J, Kusch C, Beck S, Popp M, Vogtle T, Meub M, et al. BIN2 orchestrates platelet calcium signaling in thrombosis and thrombo-inflammation. J Clin Invest. 2020;130(11):6064-79.

28. Popat S, Hubner R, Houlston RS. Systematic review of microsatellite instability and colorectal cancer prognosis. Journal of clinical oncology : official journal of the American Society of Clinical Oncology. 2005;23(3):609-18.

29. Petrelli F, Ghidini M, Cabiddu M, Pezzica E, Corti D, Turati L, et al. Microsatellite Instability and Survival in Stage II Colorectal Cancer: A Systematic Review and Meta-analysis. Anticancer Res. 2019;39(12):6431-41. 
30. Le DT, Uram JN, Wang H, Bartlett BR, Kemberling H, Eyring AD, et al. PD-1 Blockade in Tumors with Mismatch-Repair Deficiency. N Engl J Med. 2015;372(26):2509-20.

31. Naganuma A, Sakuda T, Murakami T, Aihara K, Watanuki Y, Suzuki Y, et al. Microsatellite Instabilityhigh Intrahepatic Cholangiocarcinoma with Portal Vein Tumor Thrombosis Successfully Treated with Pembrolizumab. Intern Med. 2020;59(18):2261-7.

32. Van Wyk HC, Foulis AK, Roxburgh CS, Orange C, Horgan PG, McMillan DC. Comparison of Methods to Identify Lymphatic and Blood Vessel Invasion and their Prognostic Value in Patients with Primary Operable Colorectal Cancer. Anticancer Res. 2015;35(12):6457-63.

33. Morgantetti G, Ng KL, Samaratunga H, Rhee H, Gobe GC, Wood ST. Prostate specific membrane antigen (PSMA) expression in vena cava tumour thrombi of clear cell renal cell carcinoma suggests a role for PSMA-driven tumour neoangiogenesis. Transl Androl Urol. 2019;8(Suppl 2):S147-S55.

34. Meehan M, Melvin A, Gallagher E, Smith J, McGoldrick A, Moss C, et al. Alpha-T-catenin (CTNNA3) displays tumour specific monoallelic expression in urothelial carcinoma of the bladder. Genes Chromosomes Cancer. 2007;46(6):587-93.

35. He B, Li T, Guan L, Liu FE, Chen XM, Zhao J, et al. CTNNA3 is a tumor suppressor in hepatocellular carcinomas and is inhibited by miR-425. Oncotarget. 2016;7(7):8078-89.

36. Piard J, Hu JH, Campeau PM, Rzonca S, Van Esch H, Vincent E, et al. FRMPD4 mutations cause Xlinked intellectual disability and disrupt dendritic spine morphogenesis. Hum Mol Genet. 2018;27(4):589-600.

\section{Figures}




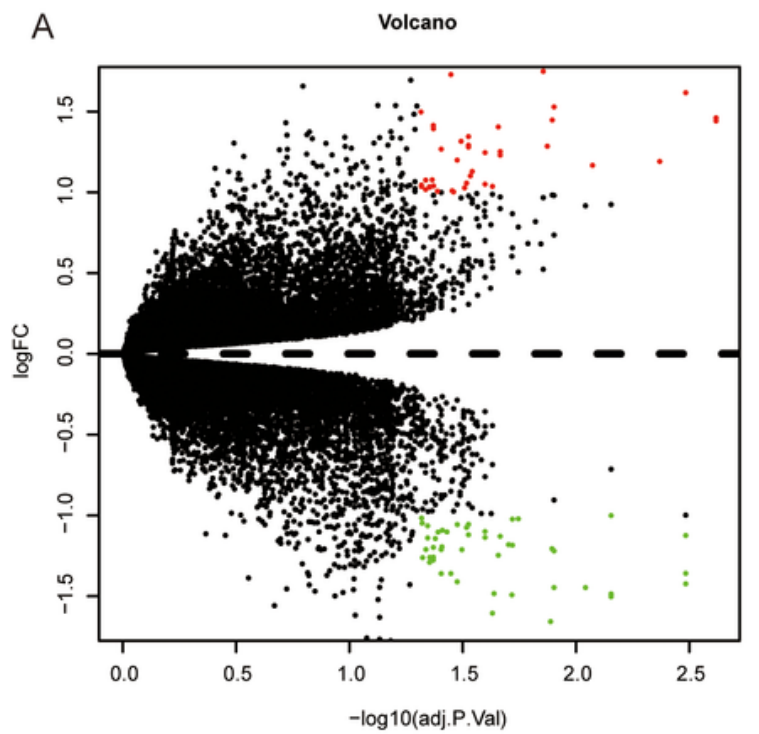

B
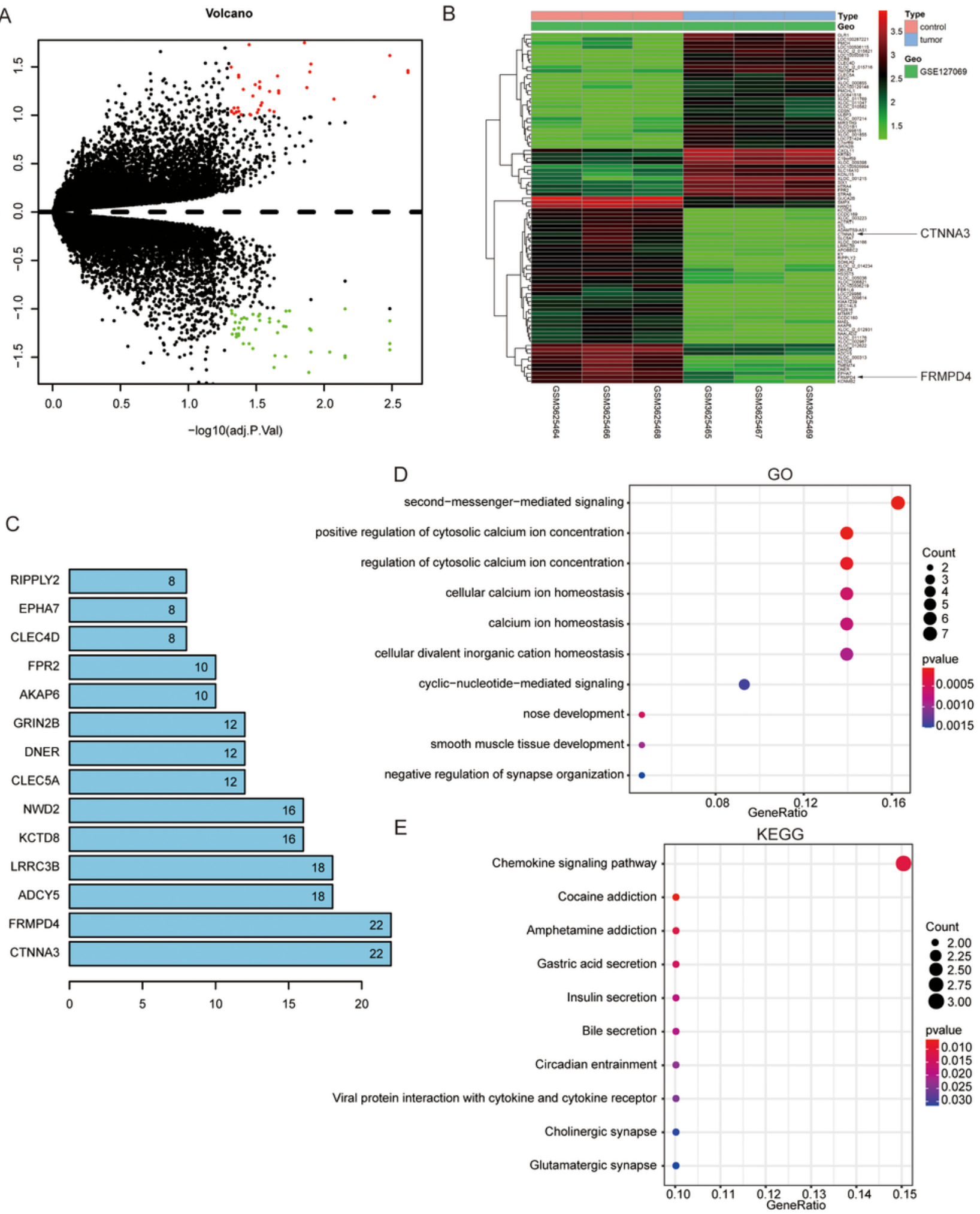

\section{Figure 1}

Differentially expressed genes were selected from GSE127069 datasets. (A) Visualization of differentially expressed genes in volcano maps. (B) The heatmap shown differential gene expression between adjacent and embolus tissues of colon cancer. (C) Analysis of PPI protein interaction network nodes. (D, E) The GO and KEGG analysis results shown that 88 differentially expressed genes were related to calcium ion, chemokine signaling pathway, etc. 


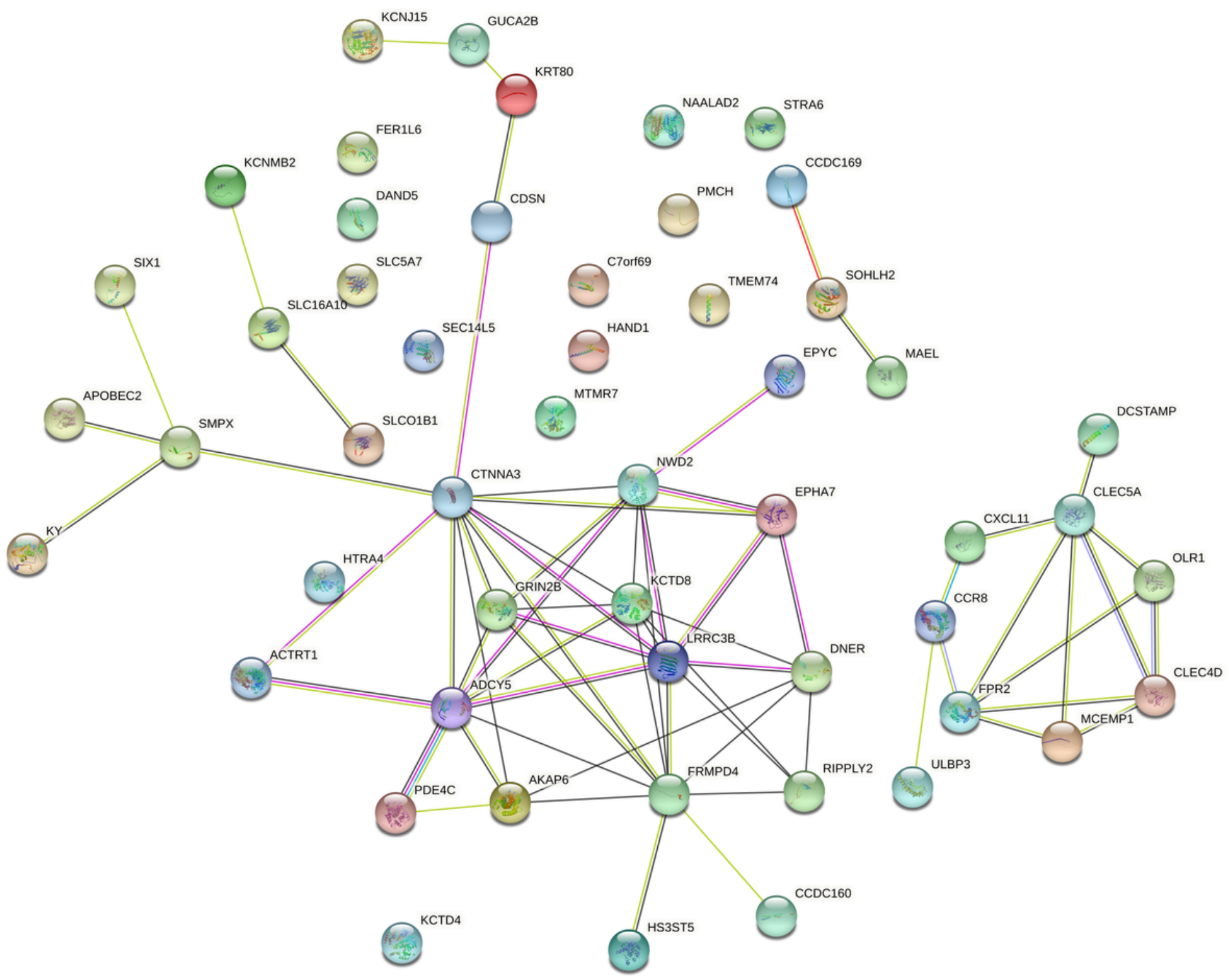

Figure 2

The PPI network of differentially expressed genes. 
A

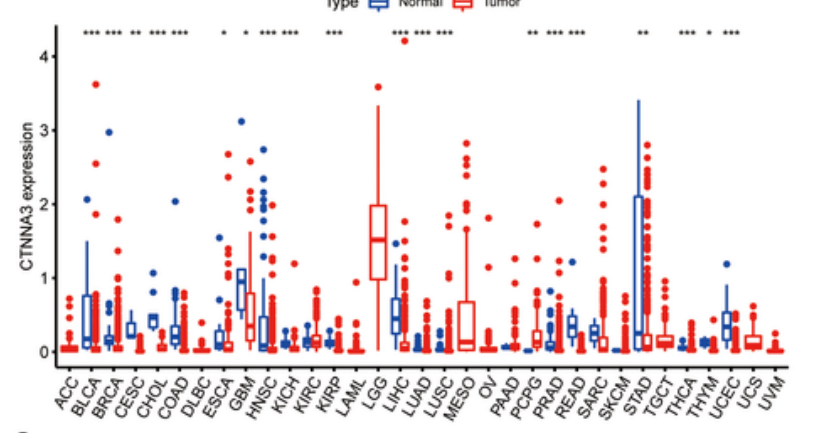

C

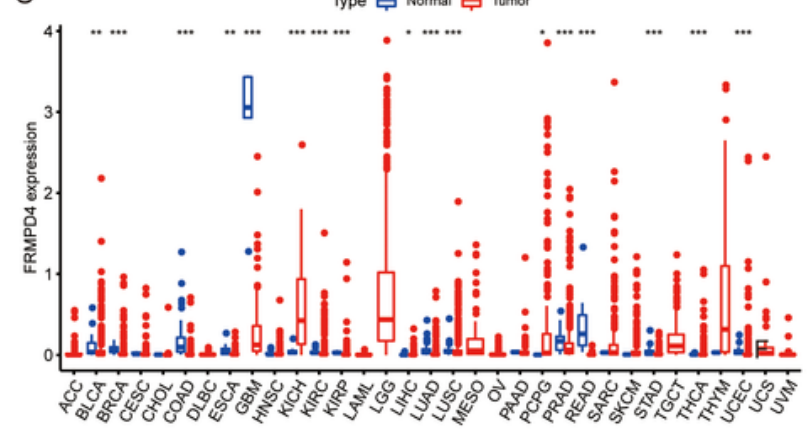

E

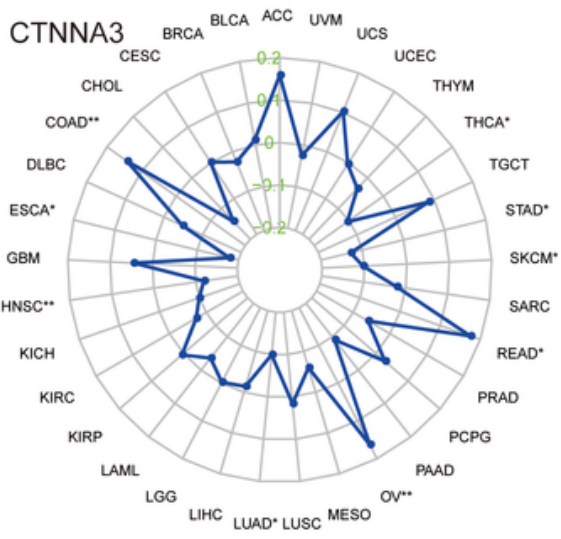

G

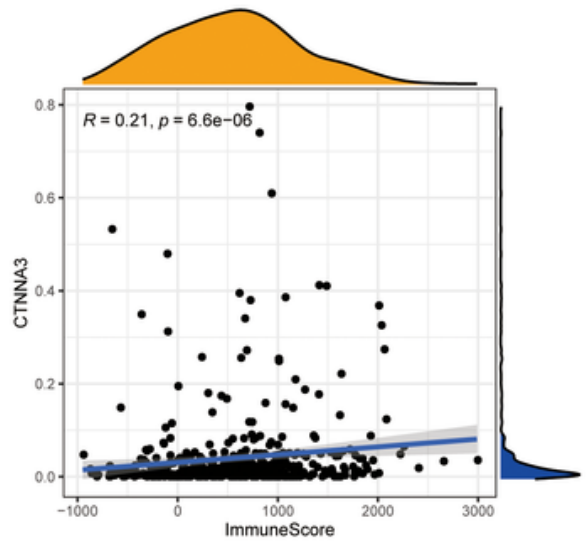

D

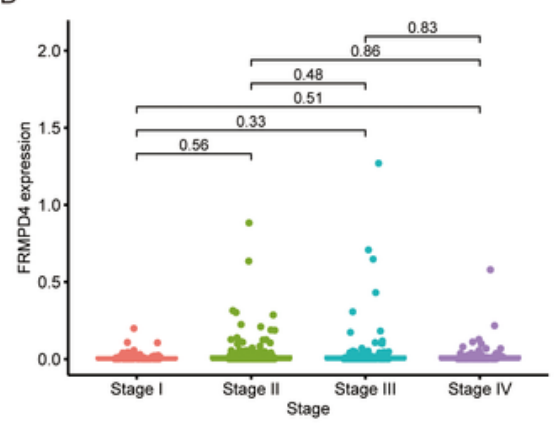

B

Stage 申 Stage I 申 Stage II 申 Stage III 申 Stage IV

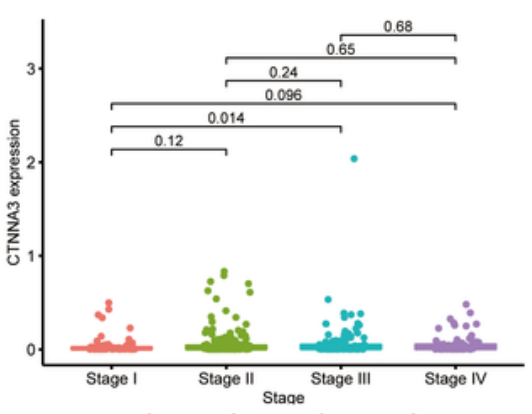

F
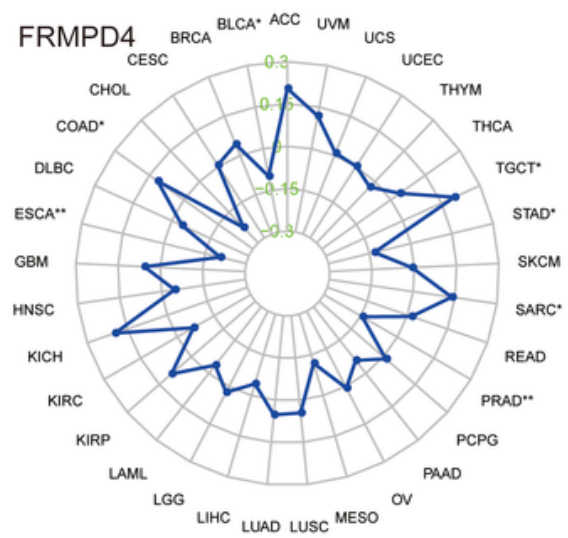

$\mathrm{H}$

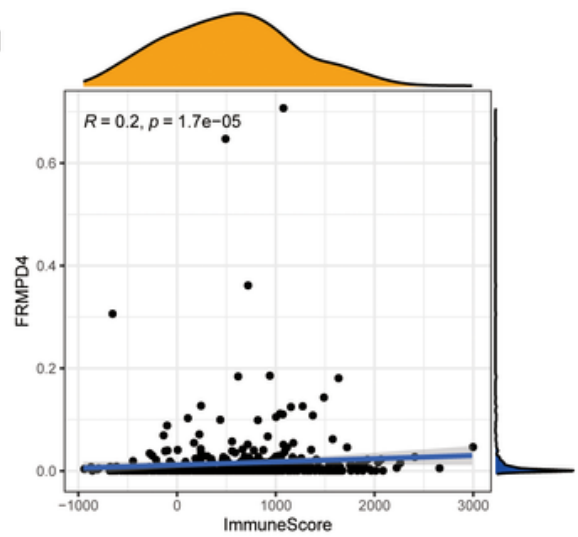

Figure 3

Pan-cancer analysis of CTNNA3 and FRMPD4. (A, C) The expression of CTNNA3 and FRMPD4 in 33

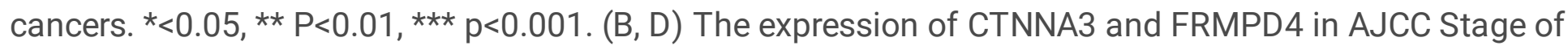
colon cancer. (E, F) The relationship between CTNNA3 and FRMPD4 and MSI in 33 cancers. $(G, H)$ The relationship between CTNNA3 and FRMPD4 and tumor immune microenvironment in colon cancer. MSI, Microsatellite instability. 


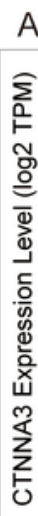
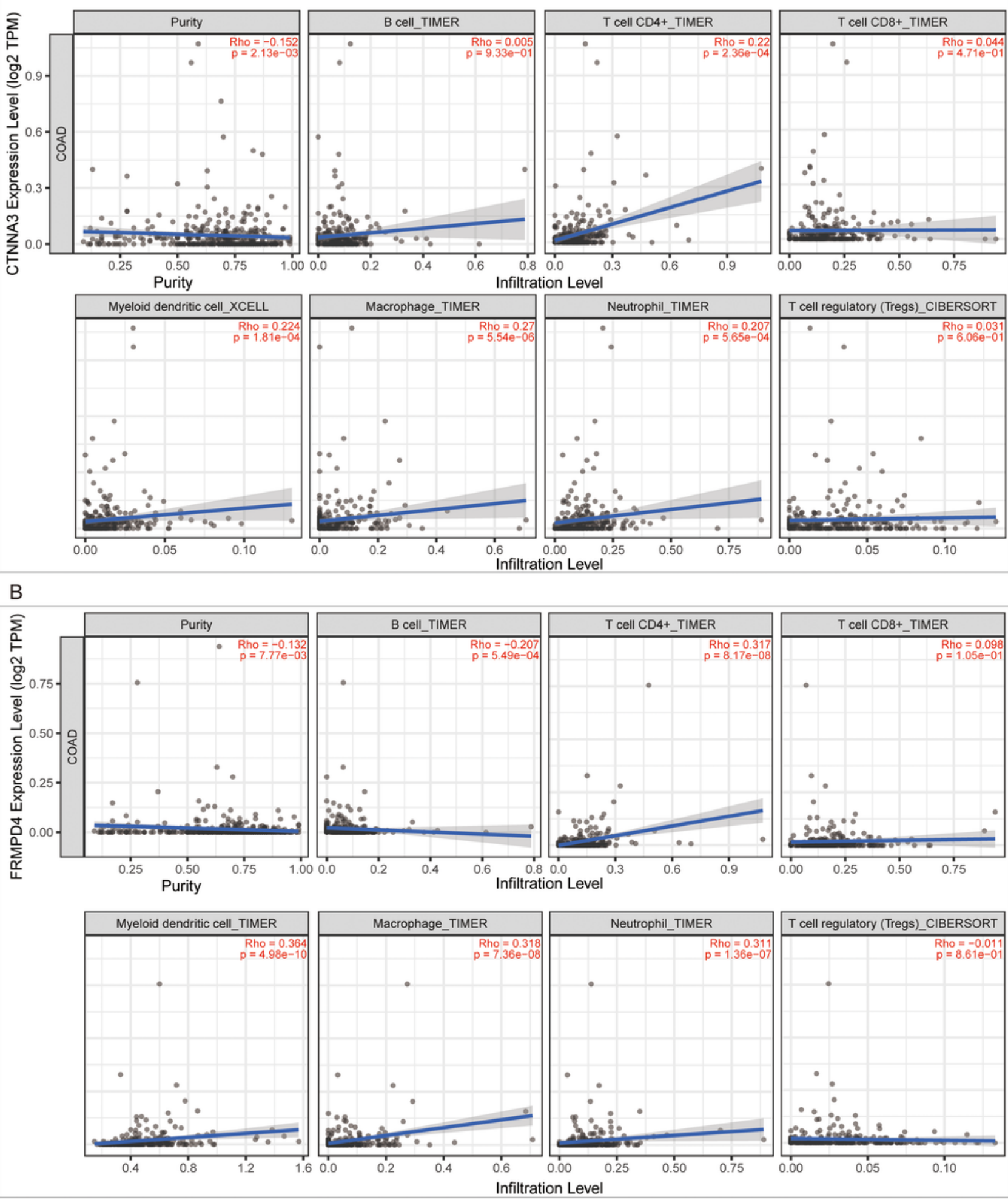

\section{Figure 4}

Analysis of CTNNA3, FRMPD4 and Immune Cell Subtype Infiltration. (A) The correlation coefficients between expression of CTNNA3 and Purity, B cells, CD4 + T cells, CD8 + T cells, DCs, macrophages, neutrophils, and Tregs, and CTNNA3 were $-0.152,0.005,0.22,0.044,0.224,0.27,0.207$, and 0.031 , respectively. (B) The correlation coefficients between expression of CTNNA3 and Purity, B cells, CD4 + T 
cells, CD8 + T cells, DCs, macrophages, neutrophils, and Tregs, and FRMPD4 were $-0.132,-0.207,0.317$, $0.098,0.364,0.318,0.311$, and -0.011 , respectively.

\section{A}

\# Samples per Patient

Profiled in Mutations

Profiled in Putative copy-number alterations from GISTIC

Vascular invasion indicator

CTNNA3

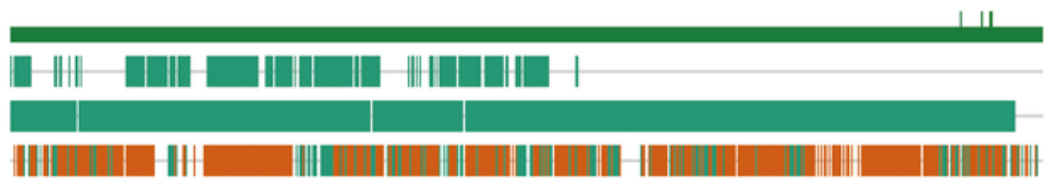
$2 \mathrm{k} \cdot \mathrm{F}$

Genetic Alteration

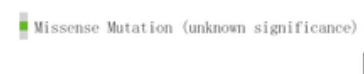

\# Samples per Patient

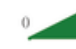

Profiled in Mutations

| ves - so

Profiled in Putative copy-number

alterations from GISTIC

.

Vascular invasion indicator

| 100 |

C
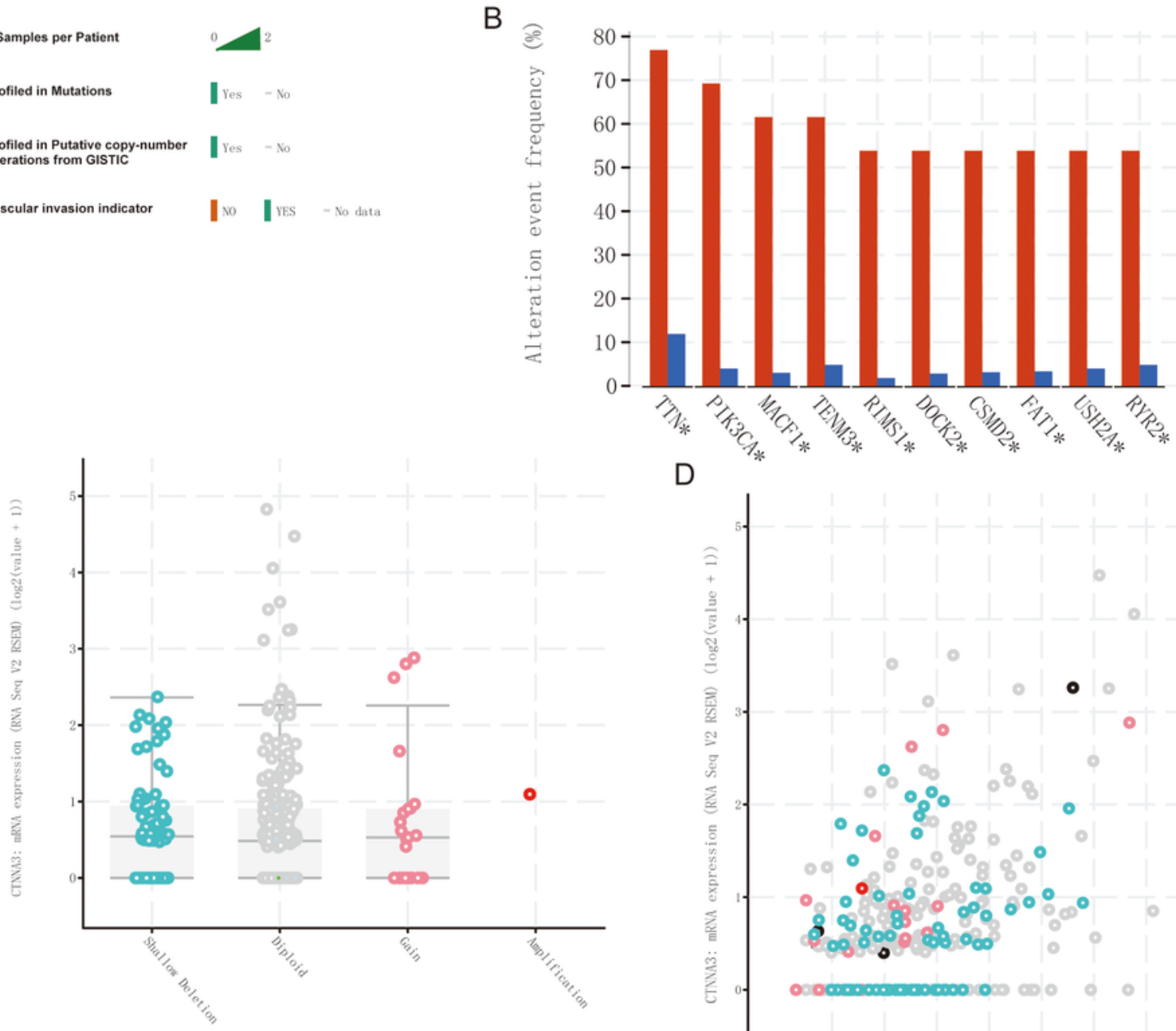

Deep Deletion

|No alterations - Not profiled

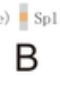

$\mathrm{D}$

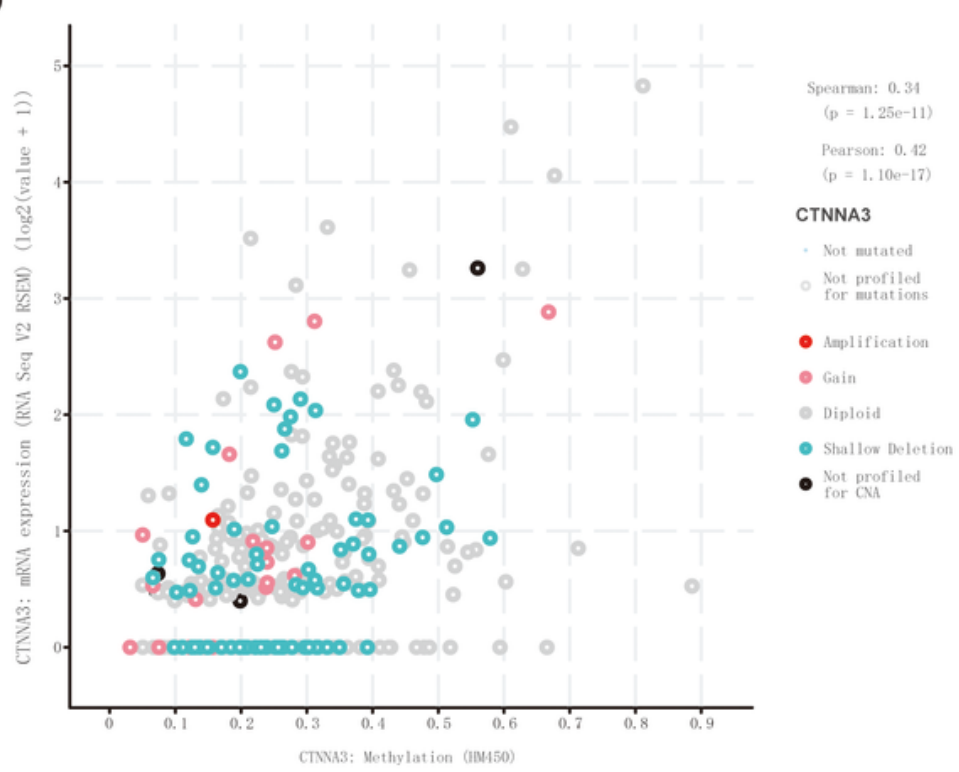

E

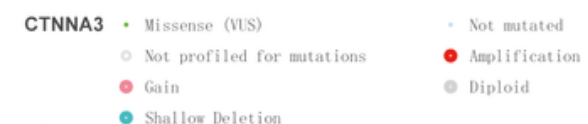

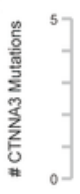

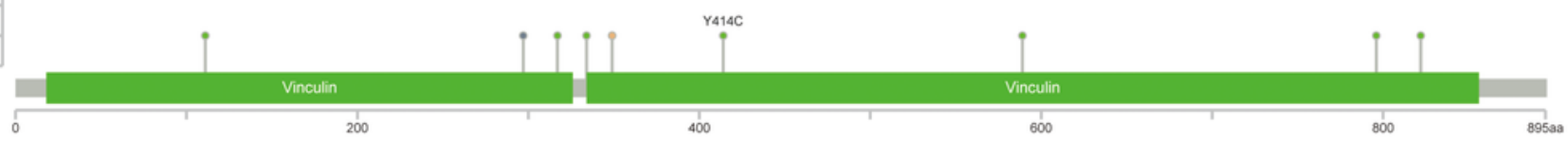

Figure 5

Analysis of gene characteristics of CTNNA3. (A) CTNNA3 gene mutation distribution and its relationship with markers of vascular infiltration. (B) Genes related to genetic changes in CTNNA3. (C) The 
relationship between CTNNA3 mRNA expression and copy number variation. (D) The relationship between CTNNA3 mRNA expression and methylation (HM450). (E) The mutation site of CTNNA3. A

\# Samples per Patient

Profiled in Mutations

Profiled in Putative copy-number alterations from GISTIC

Vascular invasion indicator

FRMPD4

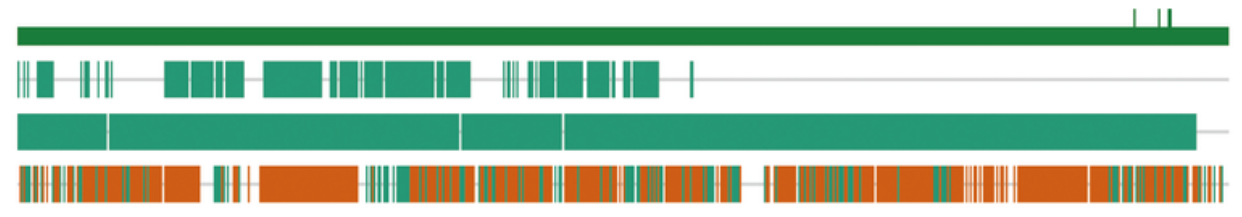

Genetic Alteration

WIissense Mutation (unknown significance) II Spl ice Mutation (unknomn

\# Samples per Patient

Profiled in Mutations

| Yes $-\mathrm{No}$

Profiled in Putative copy-number $\$ Yes - No
alterations from GISTIC

alterations from GISTIC

Vascular invasion indicator

\No \YES - No data

B

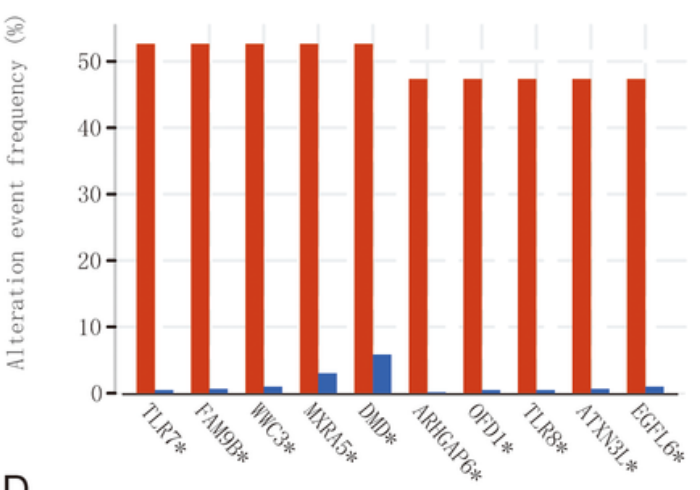

- Altered group

- Unaltered group

D

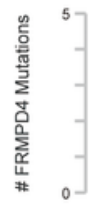

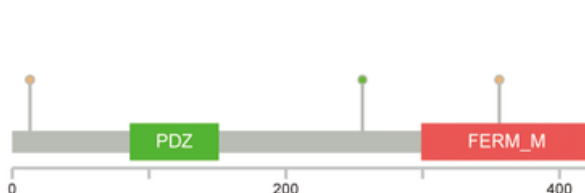

Impli ification |

Deep Deletion

No alterations - Not profiled

C

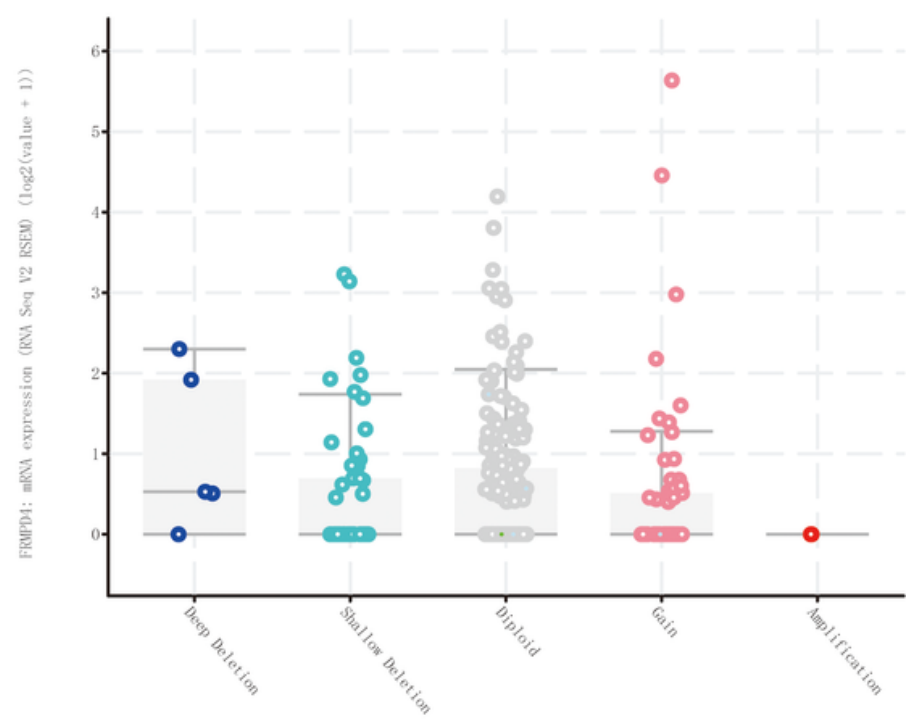

FRMPDA: Putative copy-number alterations from GISTIC

FRMPD4 - Missense (VIS) - Not mutated

- Amplification

- Shallow Deletion

- Gain

- Deep Deletion

Dor profled for mutations Diploid

Figure 6

Analysis of gene characteristics of FRMPD4. (A) FRMPD4 gene mutation distribution and its relationship with markers of vascular infiltration. (B) Genes related to genetic changes in FRMPD4. (C) The relationship between FRMPD4 mRNA expression and copy number variation. (D) The mutation site of FRMPD4. 


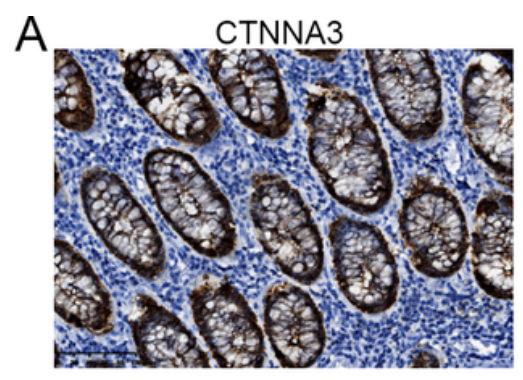

Normal

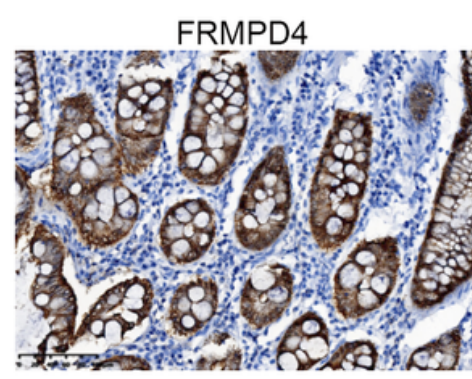

$\mathrm{B}$

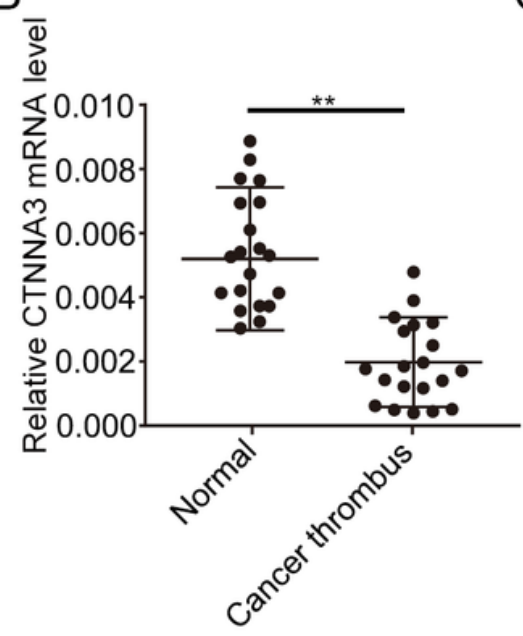

HE
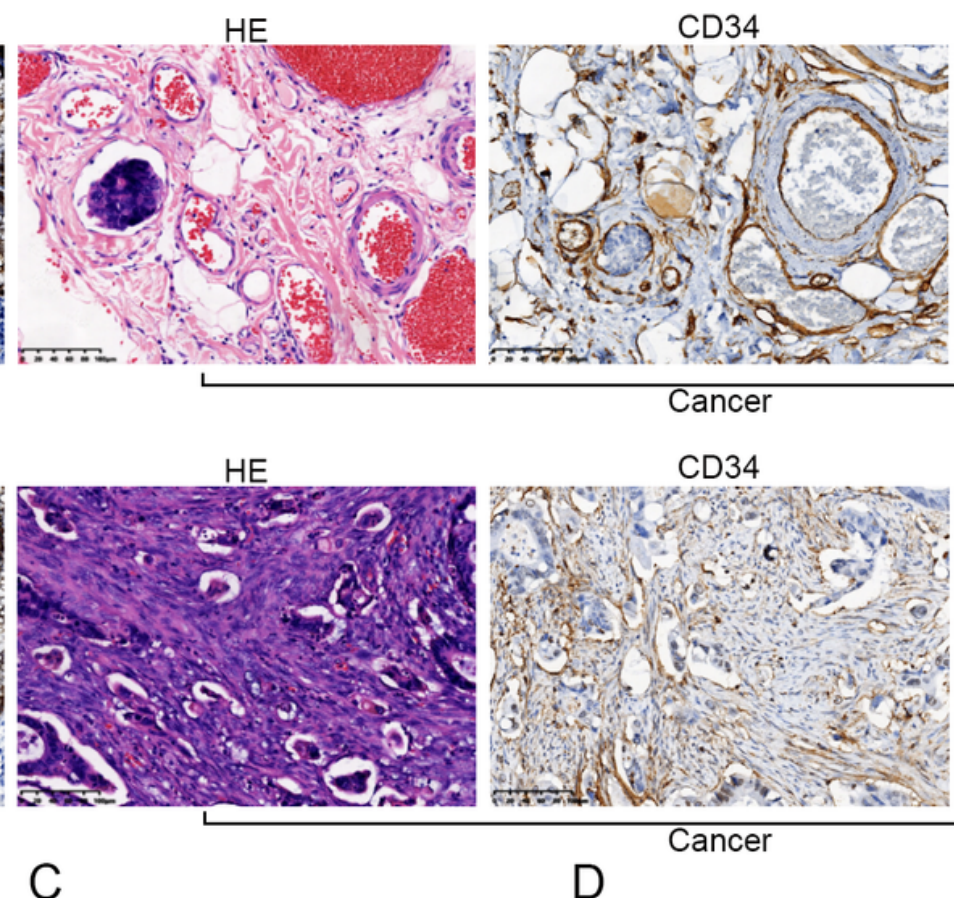

$\mathrm{D}$

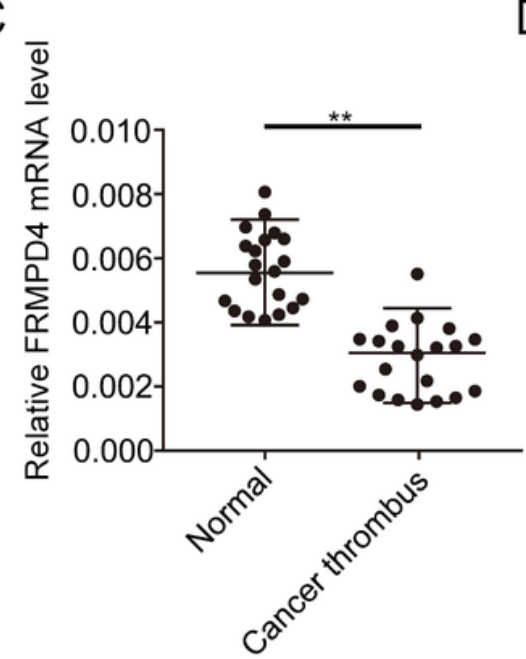

Cancer

CD34

CTNNA3

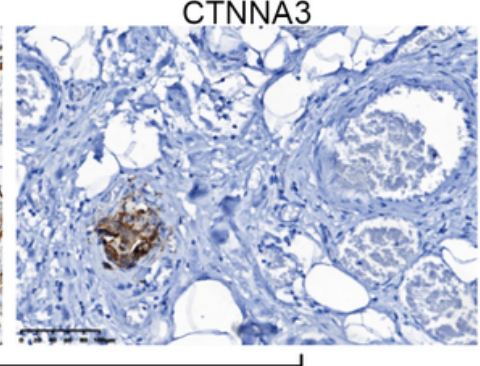

FRMPD4

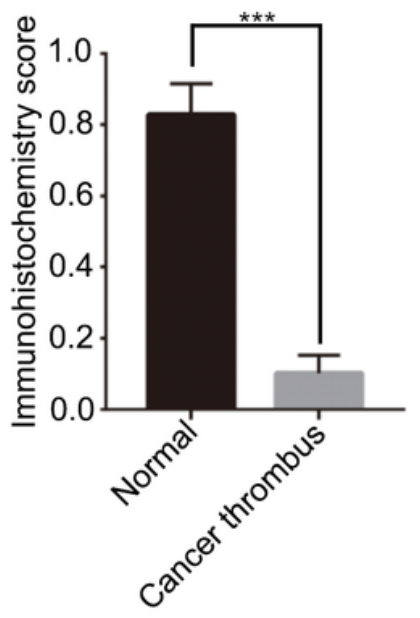

$\mathrm{E}$

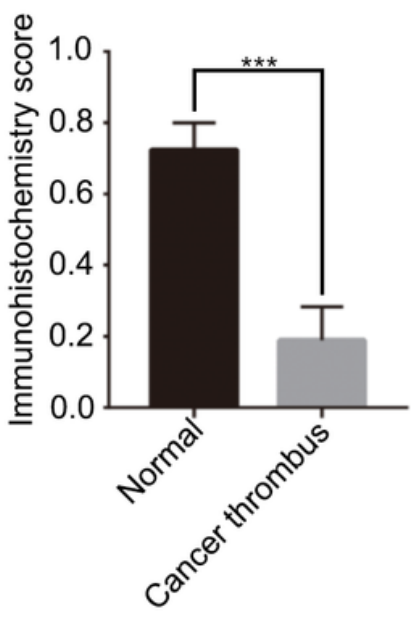

Figure 7

Expression of CTNNA3 and FRMPD4 in patients with colon cancer thrombus. (A) Immunohistochemical staining (CTNNA3, FRMPD4, CD34) and HE staining of normal tissues adjacent to colon cancer and tumor thrombus $(\times 200)$. (B, C) CTNNA3 and FRMPD4 mRNA expression in normal tissues and tumor thrombus adjacent to colon cancer. (D, E) Immunohistochemical scores of normal tissue adjacent to colon cancer and tumor thrombus. Data are presented as mean \pm standard deviation, $* \star P<0.01$, $* \star \star$ $p<0.001$. 